\title{
Normalization of enzyme expression and activity regulating vitamin A metabolism increases RAR- beta expression and reduces cellular migration in diseases caused by tuberous sclerosis gene mutations
}

ElHusseiny MM Abdelwahab

Pecsi Tudomanyegyetem

Judit Bovari-Biri

Pecsi Tudomanyegyetem

Gabor Smuk

Pecsi Tudomanyegyetem

Tunde Harko

Semmelweis Egyetem

Janos Fillinger

Semmelweis Egyetem

Judit Moldvay

Semmelweis Egyetem

Vera Krymskaya

University of Pennsylvania Perelman School of Medicine

Judit E Pongracz ( $\square$ pongracz.e.judit@pte.hu )

Pecsi Tudomanyegyetem https://orcid.org/0000-0002-0278-5556

\section{Rapid communication}

Keywords: tuberous sclerosis gene mutation, RAR $\beta$, vitamin A metabolism, retinoic acid, rapamycin

Posted Date: July 16th, 2020

DOI: https://doi.org/10.21203/rs.3.rs-41687/v1

License: (c) (i) This work is licensed under a Creative Commons Attribution 4.0 International License.

Read Full License 


\section{Abstract \\ Background}

Mutation in a tuberous sclerosis gene (TSC1 or 2) leads to continuous activation of the mammalian target of rapamycin (mTOR). mTOR activation alters various cellular functions including cellular metabolism. In association with altered metabolism, several studies have shown reduced retinoic acid receptor beta (RAR $\beta$ ) expression. Investigation of the altered metabolic process can offer the identification of novel therapeutic targets and therapeutic strategies in diseases caused by TSC mutation with limited treatment options.

\section{Methods}

RAR $\beta$ expression, metabolic enzymes expression and activity were assessed in human kidney angiomyolipoma cell line, primary lymphangioleiomyomatosis (LAM) tissue derived LAM cell lines as well as primary LAM lung tissue sections. TaqMan arrays, enzyme activities, qRT-PCRs, immunohistochemistry, immunofluorescent staining, Western blotting and metabolic enzyme regulating miRNAs were analysed. The functional effects of retinoic acid (RA) and rapamycin were tested in a scratch assay and in a 3D aggregate tissue system to assess the ability of cell migration.

\section{Results}

Metabolic enzyme arrays revealed a general deregulation of many enzymes involved in vitamin A metabolism including aldehyde dehydrogenases (ALDHs), alcohol dehydrogenases (ADHs) and Cytochrome P450 2E1 (CYP2E1). Furthermore, RAR $\beta$ downregulation was a characteristic feature of all TSC-deficient cells and tissues. Combination of the two FDA approved drugs -RA for acute myeloid leukaemia and rapamycin for TSC mutation- normalised metabolic enzyme activity, restored RAR $\beta$ expression and reduced cellular migration.

\section{Conclusion}

Deregulation of vitamin A metabolizing enzymes is a feature of TSC mutation. RA can normalize RAR $\beta$ levels and limit migration. Combination of RA with reduced dose of rapamycin might be able to decrease adverse effects of rapamycin offering an alternative treatment for patients with TSC mutation.

\section{Background}

Tuberous sclerosis, angiomyolipoma and lymphangioleiomyomatosis (LAM) are diseases characterised by slow growing tumours that are affecting many parts of the body [1] [2] including the skin, brain, kidneys and the lungs. They are caused by the mutation of tumour suppressor genes tuberous sclerosis 1 
or 2 (TSC1 or TSC2) [1]. The above tumours were initially considered benign, but angiomyolipoma and LAM have recently been reclassified as "low grade, destructive, metastasizing neoplasms". Diseases caused by TSC mutations bear the hallmarks of cancers including genetic mutations, evasion of growth suppression, resistance to cell death, metabolic reprogramming to avoid immune detection, and capability of invasion [3]. Loss of TSC activity results in continuous activation of the mTOR pathway, which is also characteristic to various neoplasms where upstream mutations or signalling malfunction have the same mTOR activating result [4]. mTOR activation alters various cellular functions including cellular proliferation, autophagy, mitochondrial biogenesis and cellular metabolism. Activation of the mTOR pathway can change anabolic cell growth processes such as protein and lipid synthesis in correlation with external growth factor or nutrient intake [5]. In our initial study of LAM, apart from detecting morphological abnormalities in mitochondria and suppression of ROS production, we identified downregulation of the proliferation suppressor nuclear receptors of the retinoic acid gene family RAR and RXR as well as several miRNA-s that regulate RAR expression including miR29b [6]. Receptors of retinoic acid (RA), a metabolite of vitamin $A$, are grouped into two distinct classes: retinoic acid receptors (RAR) and retinoid $x$ receptors (RXR) [7]. Both classes of nuclear receptors have three subtypes $(a, \beta$, and $\gamma)$ and in-patient derived LAM cell lines RAR $\beta$ mRNA expression was found significantly reduced [6]. Interestingly, RAR $\beta$ is the receptor that was associated with the anti-tumour effects of RA [8] [9] [10]. In many neoplastic diseases, expression of RAR $\beta$ is often downregulated or lost indicating that RAR $\beta$ plays an important role in tumour suppression [11]. RA (300 Da) is a metabolite of the lipophilic vitamin A (retinol) which is obtained from plant or animal sources in the form of carotenoids and retinyl esters, respectively [12]. RA is a lipophilic molecule with three isoforms: all-trans, 9-cis and 13-cis RA and is stored in forms of retinyl esters primarily in the liver as well as the kidneys, lungs and the bone marrow [12] [13]. In circulation, retinol is bound to retinol-binding protein (RBP) which enters the cells through RBP receptors (STRA6) [12]. In the cells, retinol- dehydrogenase (RDH) or alcohol-dehydrogenase (ADH) oxidize retinol to retinal which is irreversibly converted to RA by the aldehyde dehydrogenase (ALDH) family also known as retinaldehyde dehydrogenase (RALDH) [12]. RA binds to cellular retinoic acid-binding protein (CRABP) in the cell that carries RA into the nucleus where it binds to nuclear RARs to function as transcription factors. RA signalling is dependent upon its nuclear availability, controlled among others by RBP1, which is the carrier protein involved in the transport of retinol from the storage site to peripheral tissues [12]. Interestingly, the administration of RA not only activates the transcription factor RAR $\beta$ by directly binding to the transcription factor that releases co-repressors from promoters and recruits co-activators, but also increases expression of the RAR $\beta$ gene as well [14] [15]. Investigation of the above metabolic processes in tissues affected by TSC mutation where RAR $\beta$ expression is low, might offer an opportunity to identify novel therapeutic targets and treatment strategies [16]. Some additional or alternative therapies might be particularly important, as currently the only FDA approved drug to treat angiomyolipoma, tuberous sclerosis or LAM is rapamycin. Although rapamycin can slow down disease progression it cannot offer a cure. Additionally, rapamycin has significant side effects that not all patients can tolerate which results in the discontinuation of the treatment [17] [18]. Discontinuation of rapamycin, however, leads to rapid disease progression as there is no alternative treatment to be offered [18]. 
The aim of the present investigation was to reveal the metabolic changes associated TSC deficiency that lead to RARß downregulation in diseases tuberous sclerosis, angiomyolipoma and LAM [6] [19] [20] [21]. Testing the therapeutic significance of the findings were also our goal in the study.

\section{Materials And Methods \\ Ethical Statement}

LAM tissue samples were obtained from lung transplant donors for generation of cell lines, in accordance with the Declaration of Helsinki, approved by the Institutional Review Board at the University of Pennsylvania [22] and provided by the National Disease Research Interchange (NDRI, Philadelphia, PA). LAM patients had given written informed consent and all the collected samples were treated anonymously. Paraffin embedded tissue samples were obtained retrospectively from the Departments of Pathology at Semmelweis University, Budapest, and from the University of Pecs, Pecs, Hungary and the National Koranyi Institute of Pulmonology, Budapest, Hungary. The study was approved by the Medical Research Council of Hungary (54034-4/2018/EKU).

\section{LAM cell lines, bronchial smooth muscle cells (BSMC), normal human lung fibroblast (NHLF) S102 and S103 cell lines and cell culture conditions}

Primary cultures of human LAM cells where established in the Department of Medicine, University of Pennsylvania, Pennsylvania, USA [22]. Briefly, the primary cultures of LAM cells were dissociated from the LAM nodules of transplant patients. Each LAM nodule was used to establish individual cell lines (characterized based on alpha smooth muscle actin (a-SMA) expression, mTORC1 activation, HMB45 immunoreactivity, DNA synthesis, and cell migration) [23]. In the current study, four patient-derived individual LAM cell lines were used including LAM-100, LAM-111C, LAM-D9065 and LAM-HUP. As controls, primary cultures of normal, human bronchial smooth muscle cells (BSMC) and normal human lung fibroblasts (NHLF), were purchased from Lonza (Basel, Switzerland). Normal, BSMC and LAM cells were cultured at $37^{\circ} \mathrm{C}, 5 \% \mathrm{CO}_{2}$ in SMC Growth Medium (insulin, hFGF, GA, FBS and hEGF) (Lonza, Basel, Switzerland). The 621-101 cells were derived from an angiomyolipoma and carried bi-allelic inactivation of the TSC2 gene [24, 25]. The 621-102 cell line was generated by introduction of E6/E7 (pLXSN 16E6E7neo) and human telomerase (pLXSN hTERT-hyg) into a primary culture of TSC2 null human angiomyolipoma cells [26]. 621-103 was generated by stable transfection of TRI102 with wild-type TSC2 (pcDNA3.1 TSC2-zeo) into 621-101 cells [24].

\section{Haematoxylin eosin staining}


$5 \mu \mathrm{m}$ thick tissue sections were stained in Mayer's haematoxylin solution (Sigma-Aldrich, St. Louis, USA) for $10 \mathrm{~min}$, washed, then differentiated with $0.25 \%$ acetic acid and in eosin solution. Sections were mounted using Vectashield mounting medium (Vector Laboratories, Burlingame, USA). Images were taken using Nikon Eclipse Ti-U inverted microscope.

\section{Immunofluorescent staining}

Normal, BSMC, NHLF, LAM, S103 and S102 cells were cultured for 3 days using Falcon ${ }^{\text {TM }}$ chambered cell culture slides (Thermo Fisher Scientific, Waltham, USA). Cell cultures were then fixed with $4 \%$ formaldehyde and permeabilized with PBS containing 0.1\% Triton-X and 5\% BSA. Slides were incubated with primary antibodies Anti-alpha -Smooth Muscle Actin (MAB1420), Anti-mTOR Antibody (ab25880), Anti-p70 S6 kinase Antibody (Ab32529) and Anti-RAR beta (ab25880) overnight at $4^{\circ} \mathrm{C}$. Slides were washed with TBS for three times then incubated with corresponding secondary antibody: Anti-mouse Alexa 488 (A28175 1:200), Anti-rabbit Alexa 647 (A27040 1:200), Anti-rabbit Alexa 488 (A11034 1:200) or Anti-mouse Alexa 647 (A32728 1:200), for 960 min at RT. Nuclei were counter stained with DAPI. Images were acquired using an Olympus IX-81 (OLYMPUS Corporation, Tokyo, Japan) both light and fluorescence microscope.

\section{Immunohistochemistry}

$5 \mu \mathrm{m}$ thick tissue sections were stained using immunohistochemistry. First, the slides were rinsed in heated xylene and were washed with a descending series of alcohol to remove paraffin. After deparaffination the slides were rehydrated in distilled water and antigen retrieval was performed by heating the slides in Target Retrieval Solution ( $\mathrm{pH}$ 6, DAKO, Produktionsvej, Denmark) at $97^{\circ} \mathrm{C}$ for $20-30$ min. Subsequently slides were washed in $\mathrm{dH}_{2} \mathrm{O}$ and endogenous peroxidase activity was blocked with $3 \%$ $\mathrm{H}_{2} \mathrm{O}_{2}$ containing TBS ( $\mathrm{pH} 7.4$ ) for $15 \mathrm{~min}$. Then slides were washed three times with TBS containing Tween $(0.05 \%$, pH 7.4). Pre-blocking was carried out with 3\% BSA in TBS for 20 min before overnight incubation with anti- Melanoma gp100 antibody (HMB-45) (1:100, HMB-45 mouse monoclonal antibody clone: Ab787, ABcam) and anti-RAR $\beta$ (1:100, anti-RAR $\beta$ rabbit monoclonal antibody clone: Ab124701, ABcam) primary antibody at $4^{\circ} \mathrm{C}$. Following incubation slides were washed with TBS for three times then incubated with peroxidase conjugated secondary antibody (1:100, Polyclonal Goat Anti-Rabbit lgG, DAKO) for 90 min. Antibody labelling was visualized with the help of liquid DAB Substrate Chromogen System (DAKO). For nuclear counterstaining, haematoxylin staining was performed. Finally, slides were mounted with Faramount Aqueous Mounting Medium (DAKO, Produktionsvej, Denmark). Histological evaluation was performed with the help of Panoramic MIDI digital slide scanner (3DHistech, Budapest, Hungary). Image analysis was performed using ImageJ software with IHC toolbox plug-in.

\section{Rapamycin and Retinoic acid (RA) treatments}


BSMC, NHLF, LAM, S103 and S102 cell cultures were treated with mono rapamycin or RA treatments and in combined treatments using the following concentrations: 10 or 20 nM Rapamycin catalogue: tlrl-rap (InvivoGen, San Diego, USA) and 1 or $2 \mu \mathrm{M}$ retinoic acid (Sigma-Aldrich, St. Louis, USA) for $24 \mathrm{~h}$ at $37^{\circ} \mathrm{C}$, $5 \% \mathrm{CO}_{2}$.

\section{Western blot}

Cells were lysed in ice-cold RIPA buffer (Sigma-Aldrich, St. Louis, USA) supplemented with protease inhibitors (Roche Diagnostics, Mannheim, Germany) for 30 min on ice and centrifuged at $16,000 \times \mathrm{g}$ for 20 min at $4^{\circ} \mathrm{C}$. The supernatant was then used as the cell lysate. The protein content of each cell lysate was assessed using a Qubit protein assay kit (Thermo Scientific, Waltham, MA). $30 \mu \mathrm{g}$ of total protein was loaded onto Mini Protean gel (Bio-Rad, California, USA), then electrophoresis was followed by overnight blotting onto a nitrocellulose membrane using $10 \mathrm{~mA}$ current. The blots then were blocked in $5 \%$ non-fat skimmed milk blocking solution (Bio-Rad, California, USA) in TBS-T for $1 \mathrm{~h}$ and incubated with primary antibodies diluted 1:1000 in 2.5\% non-fat skimmed milk powder in TBS-T overnight at $4^{\circ} \mathrm{C}$. After washing with TBS-T, the blots were incubated with rabbit anti-goat/HRP diluted in $2.5 \%$ non-fat skimmed milk powder in TBS-T for $1 \mathrm{~h}$ at room temperature. The immunoreaction was developed with a chemiluminescence HRP substrate and recorded with ImageQuant LAS-4000 imager (GE Healthcare Life Sciences, USA).

\section{RNA isolation}

Total RNA was extracted from normal BSMC, NHLF and LAM cell cultures with MN NucleoSpin RNA isolation kit according to the manufacturer's protocol (Macherey-Nagel, Düren, Germany). The concentration of RNA samples was measured using NanoDrop (Thermo Fisher Scientific, Waltham, USA). Total RNA from human lung tissues were obtained using TRIzol reagent (Invitrogen, Thermo Fisher Scientific, Waltham, USA). RNA (1 $\mu \mathrm{g})$ was digested with DNase (Sigma-Aldrich, St. Louis, USA) to eliminate any DNA contamination. cDNA was synthesized with high capacity RNA to cDNA kit (Thermo Fisher Scientific, Waltham, USA). Reverse transcription was performed with random hexamer primers.

\section{Quantitative qRT-PCR}

qRT-PCR was performed using SensiFAST SYBR Green reagent (BioLine, London, UK) in an ABI StepOnePlus system. Gene expressions using sequence specific primers (S. Table 1) were analysed with StepOne software and normalized to beta-actin. Changes in gene expression were calculated according to the $2^{\text {-ddCt }}$ method.

\section{Quantstudio 12k flex}


cDNA was prepared using TaqMan miRNA reverse transcriptase kit and Megaplex RT primers Pool A and B (Thermo Fisher Scientific, Waltham, USA) according to manufacturers' protocol using 350ng-1000ng of total RNA as starting material. miRNA expression levels were performed using open array miRNA card Pool A and B and Quantstudio 12k flex (Thermo Fisher Scientific, Waltham, USA).

\section{Metabolic enzymes RT2 array}

cDNA was prepared using RT2 First Strand Kit (Qiagen, Hilden, Germany) according to manufacturers' protocol using 350ng-1000ng of total RNA as starting material. Metabolic enzymes mRNA expression levels were performed using Human Drug Metabolism: Phase I Enzymes arrays (Qiagen, Hilden, Germany), RT2 SYBR® Green qPCR Mastermix (Qiagen, Hilden, Germany) and results were acquired by Quantstudio 12k flex (Thermo Fisher Scientific, Waltham, USA).

\section{ALDH and ADH activity assay}

ALDH Activity Assay Kit (Abcam, MA, USA, ab155893) and Alcohol Dehydrogenase Assay Kit (Abcam, MA, USA, ab102533) were used to test ALDH and ADH activity of LAM and S102 compared to their controls before and after treatments. Activity of cell lysates was assessed using a detection kit and following the manufacturer's instructions. Enzyme activity induced colour changes were measured at OD450 nm with EnSpire ${ }^{\circledR}$ Multimode Plate Reader (PerkinElmer, Waltham, Massachusetts, USA). Pierce ${ }^{\mathrm{TM}}$ BCA Protein Assay Kit (Thermo Fisher Scientific, Waltham, USA) was used to measure protein content and results are presented as the fold change vs. control.

\section{Wound healing assay}

Cells were grown to $90 \%$ confluence in 24 well plates and wound gap was made by scratching the cell with rapamycin $(10 \mathrm{nM}), \mathrm{RA}(2 \mu \mathrm{M})$ and rapamycin $(10 \mathrm{nM})+\mathrm{RA}(2 \mu \mathrm{M})$ was after inducing the wound gap. The healing of the wound gap by cell migration and the centre of the gap was monitored with images taking with EVOS light microscopy (Thermo Fisher Scientific, Waltham, USA) and the gap area was quantified using ImageJ software.

\section{D co-cultures}

Normal human lung fibroblasts (NHLF) and bronchial smooth muscle cells (BSMC) were isolated from anonymous donors of different ages and sexes and were purchased from Lonza (Basel, Switzerland). All cells were cultured at $37^{\circ} \mathrm{C}$ and $5 \% \mathrm{CO} 2$ in primary cell culture media. NHLF, BSMC and LAM cell types were sub-cultured and mixed at 1:1 ratio then dispensed $3 * 105$ cells/well onto a low-attachment 96 -well U-bottom plates (Corning, New York, USA). 3D aggregates were formed as described previously (Kovacs et al., 2014). The 3D aggregate co-cultures were incubated in the presence or absence of $10 \mathrm{nM}$ rapamycin and/or $2 \mu \mathrm{M}$ RA for $24 \mathrm{~h}$, then collected into cryomold and sectioned for staining.

\section{Statistical analysis}


Unless otherwise noted, statistical analysis was performed with SPSS version 20 software. S102 and S103 data are presented as mean \pm technical error of three replicates and statistical analysis was performed using the t-test. LAM primary samples and their controls (BSMC $n=4$ and NHLF $n=4)$ data are presented as mean \pm standard error of mean (SEM), and statistical analysis was performed using the one-way ANOVA. $p<0.05$ was considered as significant.

\section{Results}

To investigate the involvement and regulation of the enzyme cascades associated with vitamin A metabolism in TSC deficient cells, human enzyme profiler arrays (RT2 PCR) were used first to compare mRNA levels of specific enzymes in the human kidney angiomyolipoma cell line S102 (TSC2-/-) and its control S103 (TSC2+/+). Out of the alcohol dehydrogenase family, four enzymes (ADH1A, ADH1B, ADH1C and ADH6) were significantly upregulated and one enzyme was down regulated (ADH4) in the mutant cell line (Figure 1A). In the aldehyde dehydrogenase family three enzymes (ALDH1A2, ALDH1A3, and ALDH3A1) were upregulated, while five enzymes were downregulated (ALDH1A1, ALDH3B1, ALDH3B2, ALDH4A1 and ALDH5A1) (Figure 1A). Additionally, analysis of the array data showed significant increase in CYP2E1 mRNA level (Figure 1A) (S Table 2). To predict the connection (expression, physical interaction, co-localization, etc) amongst the above described enzymes and RA in TSC mutant cells, an artificial intelligence analysis was performed (GeneMANIA database) [27] (Figure 1B). ADHs and ALDHs especially ADH4, ALDH1A2 and ALDH1A3- were predicted to physically interact with molecules involved in the RA metabolic process and RAR $\beta$ binding (Figure 1B). To determine whether ADHs and ALDHs are present in TSC2-deficient LAM cells, ADHs and ALDHs mRNA expression levels were quantified by qRTPCR in four patient derived LAM cell lines and normal individual primary human bronchial smooth muscle cell (BSMC) as well as primary normal human lung fibroblast cells (NHLF) as controls (S Figure 1). Just as in the angiomyolipoma cell line S102, in the LAM cell lines ADH1, ADH4 and ALDH1 A1-2-3 showed the exact same pattern of expression (Figure 1C). The mTOR activation induced downregulation of RAR $\beta$ expression [6] was confirmed at protein level by western blotting and immunofluorescent staining in patient derived LAM cell lines and the angiomyolipoma cell line S102 (Figure 1D, E). To confirm the cell line data in primary LAM lung tissues (S Figure 2), six individual lung tissue samples were stained for RAR $\beta$ protein (Figure 1F). The staining of primary LAM tissues confirmed that the lack of RAR $\beta$ expression is a feature of the TSC mutant LAM cells. Positive staining in LAM tissue sections was detected in immune cells sporadically present in the lung but not in structural elements of LAM lungs (Figure 1F).

To investigate whether miRNAs that regulate the expression of metabolic enzymes are altered in cells with TSC mutation, miRNA specific TaqMan arrays were used to analyse miRNA patterns in LAM cell lines, followed by single miRNA reactions for confirmation of the results (Figure 2A). Deregulation of several miRNAs were detected. miRNAs 199a, 125b, 215, 378 were significantly downregulated, while miRNAs 23a, 128, 146a, 146b were upregulated (Figure 2A). Downregulation of ALDH1A3 for example is detected following the overexpression of $\mathrm{miR}-125 \mathrm{a} / \mathrm{b}$ in previous studies [28] [29], therefore upregulation of ALDH1A3 in the presence of reduced miR125b and 199a is in agreement with the literature [28]. 
Reduced RAR $\beta$ and increased activity of ALDH enzyme have been previously associated with increased miR-146b-5p [30] that was also confirmed in the present study. Additionally, miR-378a-5p overexpression in a rat cardiomyocyte study was associated with ALDH2 downregulation therefore downregulation of miR-378a-5p (Figure 2A) in the present work supported upregulation of ALDH2 in our initial array (Figure 1A) [31]. Upregulation of miR-29b have been recently shown to promote cell migration in TSC2-deficient cells through RAR $\beta$ activation [19] which we repeatedly detected in our previous [6] and present experiments (S Figure 3 ). The schematic depiction of individual targets of miRNA-s in vitamin A metabolism and deregulation in the presence of TSC mutation is shown in Figure 2B.

To investigate the effect of RA, four patient derived LAM cell lines were treated with $1 \mu \mathrm{M}$ or $2 \mu \mathrm{M}$ [32] [33] $R A$, then RAR $\beta$ expression was quantified using qRT-PCR and immunofluorescent staining. Following incubation with $2 \mu \mathrm{M}$ RA, RAR $\beta$ mRNA expression was restored to normal levels within $24 \mathrm{~h}$ and protein expression was also enhanced (Figure 2C, D). Within $24 \mathrm{~h}, 2 \mu \mathrm{M}$ RA also stabilized mRNA expression levels of $A D H(1 A, 4)$ and $A L D H(1 A 1,1 A 2,1 A 3)$ (Figure 3A). Furthermore, in RA treated S102 and LAM cell lines $A D H$ and ALDH enzymes activity showed significant decrease compared to untreated controls (Figure 3A). Expression levels and enzymatic activity of metabolic enzymes were also quantified after 10 and $20 \mathrm{nM}$ rapamycin and/or $2 \mu \mathrm{M}$ RA treatments. The cultures were assessed after $24 \mathrm{~h}$ incubation. Combined treatment with $10 \mathrm{nM}$ rapamycin and $2 \mu \mathrm{M}$ RA stabilised mRNA expression of ADH4 and ALDH1A2-3 (Figure 3A). In contrast, ALDH1A1 was significantly downregulated, which is a known side effect of rapamycin [34]. RA $(2 \mu \mathrm{M})$ mono treatment normalized the expression levels of metabolic enzymes (Figure $3 \mathrm{~A}$ ). Combination treatment with $10 \mathrm{nM}$ rapamycin and $2 \mu \mathrm{M}$ RA performed better to bring mRNA levels close to normal in comparison with rapamycin mono treatment. As expected, not just expression but enzymatic activity of ALDH was also inhibited by rapamycin in both mono and combination treatment, yet lower dose of rapamycin $(10 \mathrm{nM})$ resulted in a reduced inhibitory effect (Figure 3B, C). The enzymatic activity of ADH was closely normalized when rapamycin (10 nM) and RA (2 $\mu \mathrm{M}$ ) were used in combination (Figure 3D, E).

As RA is known to control cell migration, we tested the effect of RA in combination with rapamycin on cell motility [35] [36]. In theory, the effect of rapamycin and RA combination can be synergetic in TSCdeficient cells; limiting the cell migration capability by RA and inhibiting proliferation by rapamycin [36]. To investigate whether the $10 \mathrm{nM}$ of rapamycin and $2 \mu \mathrm{M}$ RA in combination can still control cellular migration just as $20 \mathrm{nM}$ of rapamycin monotreatment can, a scratch assay was performed. The combined treatment of rapamycin $(10 \mathrm{nM})$ and RA $(2 \mu \mathrm{M})$ decreased cellular migration significantly (Figure 4A, B). Apart from increased cell migration, TSC-deficiency is characterized by structural changes of the affected tissues. Such changes cannot be detected in traditional 2D cell cultures, therefore a 3D tissue aggregates were used [37]. The 3D aggregate tissues containing LAM cells developed empty sac formations after $24 \mathrm{~h}$ incubation which feature was reduced after $10 \mathrm{nM}$ rapamycin and/or $2 \mu \mathrm{M} \mathrm{RA}$ treatment making the LAM cell containing co-cultures structurally similar to the aggregate cultures containing only TSC+/+ BSMC and NHLF cells (Figure 4C). Additionally, $10 \mathrm{nM}$ rapamycin and/or $2 \mu \mathrm{M}$ RA treatments increased RAR $\beta$ protein expression (Figure 4D). 


\section{Discussion}

In our previous study, we detected correlation between the mTOR activation and mitochondrial dysfunction in LAM, that also resulted in downregulation of the proliferation suppressor nuclear receptor family of RAR and RXR [6]. Recent studies have also shown that rapamycin treatment induced upregulation of miR-29b in LAM affected cell growth, migration, and invasion via regulation of RAR $\beta$ activity [19].

In the present study, we confirmed that downregulation of RAR $\beta$ is not just a feature of TSC-deficient cell lines (angiomyolipoma, LAM primary cell lines) but it is characteristic to LAM disease (Figure 1D, E, F). In many cancers [38] the activity of RAR $\beta$ itself is suppressed via various pathways leading up to mTOR activation $[19,39]$. As RAR $\beta$ levels are associated with alterations in the vitamin A metabolic pathway [11], clear understanding of vitamin A metabolism in connection with TSC mutation is important for better disease control.

In the present study, we used primary LAM cell lines pre-dating the rapamycin era, therefore the metabolic enzymes were not affected by rapamycin. We also used angiomyolipoma cell lines to confirm the effects of TSC mutations on retinol metabolism. Based on our study the ability to metabolise retinol is seriously compromised in TSC-deficient cells (Figure 1). Many enzymes, including ADH1A, ADH1B, ADH1C ADH6, ALDH1A2, ALDH1A3, and ALDH3A1 were drastically upregulated, while others including ADH4, ALDH1A1, ALDH3B1, ALDH3B2, ALDH4A1 and ALDH5A1 were significantly downregulated (Figure 1). The characteristic function of ALDHs is to oxidize aldehydes that would otherwise participate in signalling pathways to induce cellular, to minimize ROS production and to mediate RA signalling cascades [40]. These regulatory mechanisms are important in regulation of proliferation, tumorigenesis and resistance to therapy [41]. Expression of ALDHs are also regulated by RA compounds including the chemotherapeutic vitamin A or chemically related molecules (retinoids) as well as oncogenic pathways including the WNT/ $\beta$-catenin and the MUC1-C/ ERK pathways [40]. CYP $2 E 1$ also degrades retinoic acid (RA) and retinol to polar metabolites with toxic and apoptotic properties which can promote carcinogenesis [42]. Imbalance in alcohol dehydrogenases level acts as a competitive inhibitor of retinol oxidation in liver which may reduce the biosynthesis of retinoic acid [43]. It is especially important that co-expression and physical interactions amongst the metabolic enzymes of retinol are tightly controlled. Along at the control level, we identified significant deregulation of miRNAs that are involved in the modulation of ALDH and ADH enzyme as well as RAR $\beta$ expression.

\section{Conclusion}

Importantly, several enzymes of the vitamin A metabolism, its regulatory miRNAs, and the nuclear receptors themselves including RAR $\beta$ can become potential therapeutic targets in TSC mutant or deregulated neoplasms [44] [45]. RA for example is an FDA approved drug for acute myeloid leukaemia as RA can normalize RAR $\beta$ levels and limit cancer cell migration and therefore disease progression [45]. Based on our study we propose that clinical assessment of the combination of RA with reduced dose of 
rapamycin might reduce adverse reactions to rapamycin in tuberous sclerosis, LAM and angiomyolipoma patients. Limiting the inhibitory effect of rapamycin on ALDH allow better balance of vitamin A metabolism allowing better control of RAR $\beta$ activity. Increased expression and activity of RAR $\beta$ might lead to inhibition of cellular migration and improved disease control. Further studies are certainly required to support our in vitro results.

\section{Abbreviations}

ADHs/RDHs alcohol dehydrogenases/retinol dehydrogenases

ALDHs aldehyde dehydrogenases

ATRA all-trans retinoic acid/ tretinoin

BSMC human bronchial smooth muscle cell

CRABP cellular retinoic acid-binding protein

CYP Cytochrome P450

GFP green fluorescent protein

H\&E hematoxylin and eosin

HMB45 melanoma marker

LAM Lymphangioleiomyomatosis

mTOR Mammalian target of rapamycin

NHLF normal human lung fibroblast cell

RA Retinoic acid

RALDH retinaldehyde dehydrogenase

RAR Retinoic acid receptor

RBP retinol-binding protein

RXR retinoid $x$ receptors

S102 human female kidney angiomyolipoma cell line 621-102 TSC2-/-

S103 human female kidney angiomyolipoma cell line 621-103 TSC2+/+

STRA6 Stimulated by retinoic acid 6

TSC 1-2 Tuberous sclerosis 1-2

\section{Declarations}


All data are available from the corresponding author upon reasonable request.

\section{Acknowledgements}

We would like to thank prof. Elizabeth Petri Henske, Harvard Medical School for the angiomyolipoma cell lines.

\section{Funding}

JEP: TUDFO/51757-1/2019-ITM and GINOP 2.3.2-15-2016-00022 funds.

\section{Authors' contributions}

EMMA and JB: performed the experiments, isolated RNA and protein from NHLF, SMC and LAM, cellular staining, embedding of samples for microscopy, performed data analysis, prepared figures; KV: generated the LAM cell lines and performed experiments on angiomyolipoma cell lines; JM, JF, TH, GS: selected the samples; JEP designed the studies; EMMA and JEP have written the manuscript.

\section{Corresponding author}

Correspondence to Judit E Pongracz

\section{Ethics declarations}

\section{Ethics approval}

LAM tissue samples were obtained from lung transplant donors for generation of cell lines, in accordance with the Declaration of Helsinki, approved by the Institutional Review Board at the University of Pennsylvania [22] and provided by the National Disease Research Interchange (NDRI, Philadelphia, PA). LAM patients had given written informed consent and all the collected samples were treated anonymously. Paraffin embedded tissue samples were obtained retrospectively from the Departments of Pathology at Semmelweis University, Budapest, and from the University of Pecs, Pecs, Hungary and the National Koranyi Institute of Pulmonology, Budapest, Hungary. The study was approved by the Medical Research Council of Hungary (54034-4/2018/EKU).

\section{Consent for publication}

Not applicable

\section{Competing interests}

The authors declare that they have no competing interest.

\section{References}


1. Leung AKC, Robson WLM. Tuberous Sclerosis Complex: A Review. J Pediatr Heal Care. 2007;21:10814. doi:10.1016/j.pedhc.2006.05.004.

2. Lin $C$, Jin $L$, Yang $Y$, Ding $Y, W u X, N i$, et al. Tuberous sclerosis-associated renal angiomyolipoma: A report of two cases and review of the literature. Mol Clin Oncol. 2017;7:706-8. doi:10.3892/mco.2017.1377.

3. McCormack FX, Travis WD, Colby T V., Henske EP, Moss J. Lymphangioleiomyomatosis - Calling it what it is: A low-grade, destructive, metastasizing neoplasm. American Journal of Respiratory and Critical Care Medicine. 2012;186:1210-2. doi:10.1164/rccm.201205-08480E.

4. Hammes SR, Krymskaya VP. Targeted Approaches toward Understanding and Treating Pulmonary Lymphangioleiomyomatosis (LAM). Horm Cancer. 2013;4:70-7. doi:10.1007/s12672-012-0128-4.

5. Laplante M, Sabatini DM. mTOR signaling at a glance. J Cell Sci. 2009;122 Pt 20:3589-94. doi:10.1242/jcs.051011.

6. Abdelwahab EMM, Pal S, Kvell K, Sarosi V, Bai P, Rue R, et al. Mitochondrial dysfunction is a key determinant of the rare disease lymphangioleiomyomatosis and provides a novel therapeutic target. Oncogene. 2019;38:3093-101. doi:10.1038/s41388-018-0625-1.

7. Altucci L, Leibowitz MD, Ogilvie KM, de Lera AR, Gronemeyer H. RAR and RXR modulation in cancer and metabolic disease. Nat Rev Drug Discov. 2007;6:793-810. doi:10.1038/nrd2397.

8. Das BC, Thapa P, Karki R, Das S, Mahapatra S, Liu T-C, et al. Retinoic acid signaling pathways in development and diseases. Bioorg Med Chem. 2014;22:673-83. doi:10.1016/j.bmc.2013.11.025.

9. Li Y, Wongsiriroj N, Blaner WS. The multifaceted nature of retinoid transport and metabolism. Hepatobiliary Surg Nutr. 2014;3:126-39. doi:10.3978/j.issn.2304-3881.2014.05.04.

10. Tsuji $M$, Shudo $K$, Kagechika $H$. Identifying the receptor subtype selectivity of retinoid $X$ and retinoic acid receptors via quantum mechanics. FEBS Open Bio. 2017;7:391-6. doi:10.1002/22115463.12188.

11. Sun S-Y, Lotan R. Retinoids and their receptors in cancer development and chemoprevention. Crit Rev Oncol Hematol. 2002;41:41-55. doi:10.1016/S1040-8428(01)00144-5.

12. O’Byrne SM, Blaner WS. Retinol and retinyl esters: biochemistry and physiology. J Lipid Res. 2013;54:1731-43. doi:10.1194/jlr.R037648.

13. Chelstowska S, Widjaja-Adhi MAK, Silvaroli JA, Golczak M. Molecular Basis for Vitamin A Uptake and Storage in Vertebrates. Nutrients. 2016;8:676. doi:10.3390/nu8110676.

14. Sucov HM, Murakami KK, Evans RM. Characterization of an autoregulated response element in the mouse retinoic acid receptor type beta gene. Proc Natl Acad Sci. 1990;87:5392-6. doi:10.1073/pnas.87.14.5392.

15. Leid $M$, Kastner $P$, Chambon $P$. Multiplicity generates diversity in the retinoic acid signalling pathways. Trends Biochem Sci. 1992;17:427-33. doi:10.1016/0968-0004(92)90014-z.

16. Kishton RJ, Rathmell JC. Novel Therapeutic Targets of Tumor Metabolism. Cancer J. 2015;21:62-9. doi:10.1097/PP0.0000000000000099. 
17. Merkel S, Mogilevskaja N, Mengel M, Haller H, Schwarz A. Side Effects of Sirolimus. Transplant Proc. 2006;38:714-5.

18. Salmon AB. About-face on the metabolic side effects of rapamycin. Oncotarget. 2015;6:2585-6. doi:10.18632/oncotarget.3354.

19. Liu HJ, Lam HC, Baglini C V., Nijmeh J, Cottrill AA, Chan SY, et al. Rapamycin-upregulated miR-29b promotes mTORC1-hyperactive cell growth in TSC2-deficient cells by downregulating tumor suppressor retinoic acid receptor $\beta$ (RAR $\beta$ ). Oncogene. 2019;38:7367-83.

20. Li C, Zhou X, Sun Y, Zhang E, Mancini JD, Parkhitko A, et al. Faslodex inhibits estradiol-induced extracellular matrix dynamics and lung metastasis in a model of lymphangioleiomyomatosis. Am J Respir Cell Mol Biol. 2013;49:135-42. doi:10.1165/rcmb.2012-04760C.

21. Boorjian SA, Sheinin Y, Crispen PL, Lohse CM, Kwon ED, Leibovich BC. Hormone Receptor Expression in Renal Angiomyolipoma: Clinicopathologic Correlation. Urology. 2008;72:927-32. doi:10.1016/j.urology.2008.01.067.

22. Goncharova EA, Goncharov DA, Eszterhas A, Hunter DS, Glassberg MK, Yeung RS, et al. Tuberin regulates p70 S6 kinase activation and ribosomal protein S6 phosphorylation: A role for the TSC2 tumor suppressor gene in pulmonary lymphangioleiomyomatosis (LAM). J Biol Chem. 2002;277:30958-67.

23. Goncharova EA, Goncharov DA, Lim PN, Noonan D, Krymskaya VP. Modulation of cell migration and invasiveness by tumor suppressor TSC2 in lymphangioleiomyomatosis. Am J Respir Cell Mol Biol. 2006;34:473-80.

24. Yu J, Astrinidis A, Howard S, Henske EP. Estradiol and tamoxifen stimulate LAM-associated angiomyolipoma cell growth and activate both genomic and nongenomic signaling pathways. American Journal of Physiology - Lung Cellular and Molecular Physiology. 2004;286 4 30-4. doi:10.1152/ajplung.00204.2003.

25. Carsillo T, Astrinidis A, Henske EP. Mutations in the tuberous sclerosis complex gene TSC2 are a cause of sporadic pulmonary lymphangioleiomyomatosis. Proc Natl Acad Sci U S A. 2000;97:608590. doi:10.1073/pnas.97.11.6085.

26. Furukawa T, Duguid WP, Rosenberg L, Viallet J, Galloway DA, Tsao MS. Long-term culture and immortalization of epithelial cells from normal adult human pancreatic ducts transfected by the E6E7 gene of human papilloma virus 16. Am J Pathol. 1996;148:1763-70. /pmc/articles/PMC1861644/?report=abstract. Accessed 12 Jul 2020.

27. Mostafavi S, Ray D, Warde-Farley D, Grouios C, Morris Q. GeneMANIA: A real-time multiple association network integration algorithm for predicting gene function. Genome Biol. 2008;9 SUPPL. 1:S4. doi:10.1186/gb-2008-9-s1-s4.

28. Duan J-J, Cai J, Guo Y-F, Bian X-W, Yu S-C. ALDH1A3, a metabolic target for cancer diagnosis and therapy. Int J Cancer. 2016;139:965-75. doi:10.1002/ijc.30091.

29. Tili E, Michaille JJ, Luo Z, Volinia S, Rassenti LZ, Kipps TJ, et al. The down-regulation of miR-125b in chronic lymphocytic leukemias leads to metabolic adaptation of cells to a transformed state. Blood. 
2012;120:2631-8. doi:10.1182/blood-2012-03-415737.

30. Czajka AA, Wójcicka A, Kubiak A, Kotlarek M, Bakuła-Zalewska E, Koperski $Ł$, et al. Family of microRNA-146 Regulates RAR $\beta$ in Papillary Thyroid Carcinoma. PLoS One. 2016;11:e0151968. doi:10.1371/journal.pone.0151968.

31. Wang Z, Song J, Zhang L, Huang S, Bao L, Chen F, et al. Increased expression of microRNA-378a-5p in acute ethanol exposure of rat cardiomyocytes. Cell Stress \& Chaperones. 2017;22:245-52. doi:10.2307/44848852.

32. Cheung Y-T, Lau WK-W, Yu M-S, Lai CS-W, Yeung S-C, So K-F, et al. Effects of all-trans-retinoic acid on human SH-SY5Y neuroblastoma as in vitro model in neurotoxicity research. Neurotoxicology. 2009;30:127-35. doi:10.1016/J.NEURO.2008.11.001.

33. Geisen C, Denk C, Gremm B, Baust C, Karger A, Bollag W, et al. High-level expression of the retinoic acid receptor beta gene in normal cells of the uterine cervix is regulated by the retinoic acid receptor alpha and is abnormally down-regulated in cervical carcinoma cells. Cancer Res. 1997;57:1460-7. http://www.ncbi.nlm.nih.gov/pubmed/9108446. Accessed 30 Sep 2019.

34. Mu X, Isaac C, Schott T, Huard J, Weiss K. Rapamycin Inhibits ALDH Activity, Resistance to Oxidative Stress, and Metastatic Potential in Murine Osteosarcoma Cells. Sarcoma. 2013;2013:480713. doi:10.1155/2013/480713.

35. Flamini MI, Gauna GV, Sottile ML, Nadin BS, Sanchez AM, Vargas-Roig LM. Retinoic acid reduces migration of human breast cancer cells: Role of retinoic acid receptor beta. $\mathrm{J}$ Cell Mol Med. 2014;18:1113-23. doi:10.1111/jcmm.12256.

36. Varma S, Khandelwal RL. Effects of rapamycin on cell proliferation and phosphorylation of mTOR and p70S6K in HepG2 and HepG2 cells overexpressing constitutively active Akt/PKB. Biochim Biophys Acta - Gen Subj. 2007;1770:71-8. doi:10.1016/j.bbagen.2006.07.016.

37. Kovacs T, Csongei V, Feller D, Ernszt D, Smuk G, Sarosi V, et al. Alteration in the Wnt microenvironment directly regulates molecular events leading to pulmonary senescence. Aging Cell. 2014;13:838-49. doi:10.1111/acel.12240.

38. Valastyan S, Weinberg RA. Tumor metastasis: Molecular insights and evolving paradigms. Cell. 2011;147:275-92. doi:10.1016/j.cell.2011.09.024.

39. Agudo M, Yip P, Davies M, Bradbury E, Doherty P, McMahon S, et al. A retinoic acid receptor $\beta$ agonist (CD2019) overcomes inhibition of axonal outgrowth via phosphoinositide 3-kinase signalling in the injured adult spinal cord. Neurobiol Dis. 2010;37:147-55.

40. Clark DW, Palle K. Aldehyde dehydrogenases in cancer stem cells: Potential as therapeutic targets. Ann Transl Med. 2016;4. doi:10.21037/atm.2016.11.82.

41. Singh S, Brocker C, Koppaka V, Chen Y, Jackson BC, Matsumoto A, et al. Aldehyde dehydrogenases in cellular responses to oxidative/ electrophilicstress. Free Radical Biology and Medicine. 2013;56:89101. doi:10.1016/j.freeradbiomed.2012.11.010.

42. Orywal K, Szmitkowski M. Alcohol dehydrogenase and aldehyde dehydrogenase in malignant neoplasms. Clinical and Experimental Medicine. 2017;17:131-9. doi:10.1007/s10238-016-0408-3. 
43. Kedishvili NY. Retinoic acid synthesis and degradation. Subcell Biochem. 2016;81:127-61. doi:10.1007/978-94-024-0945-1_5.

44. Idres N, Marill J, Flexor MA, Chabot GG. Activation of retinoic acid receptor-dependent transcription by all-trans-retinoic acid metabolites and isomers. J Biol Chem. 2002;277:31491-8. doi:10.1074/jbc.M205016200.

45. Su M, Alonso S, Jones JW, Yu J, Kane MA, Jones RJ, et al. All-trans retinoic acid activity in acute myeloid leukemia: Role of cytochrome P450 enzyme expression by the microenvironment. PLoS One. 2015;10. doi:10.1371/journal.pone.0127790.

\section{Figures}

A
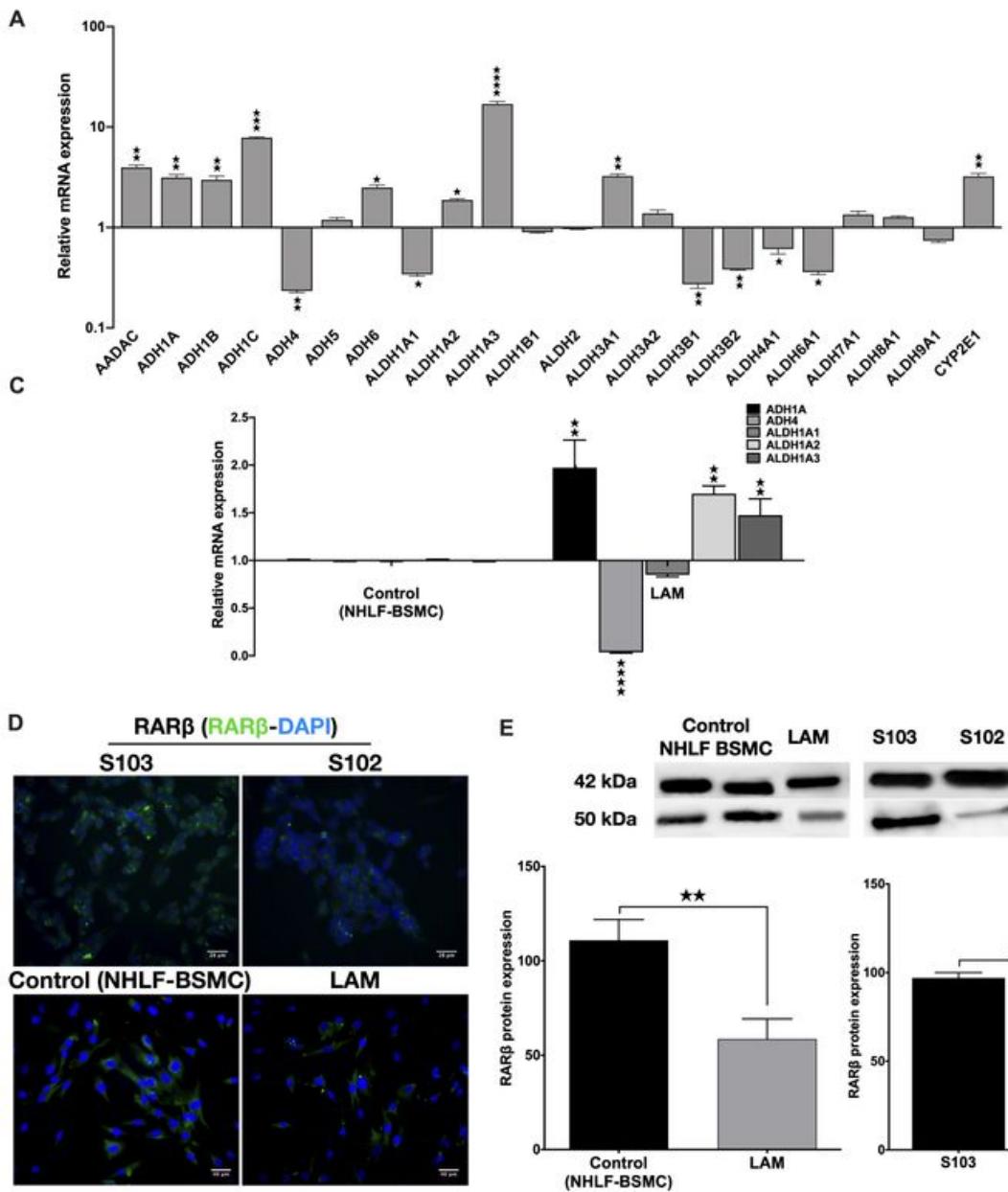

E
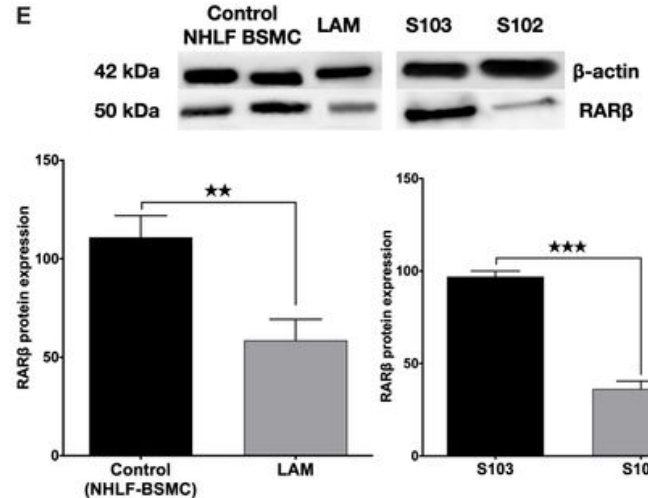

B
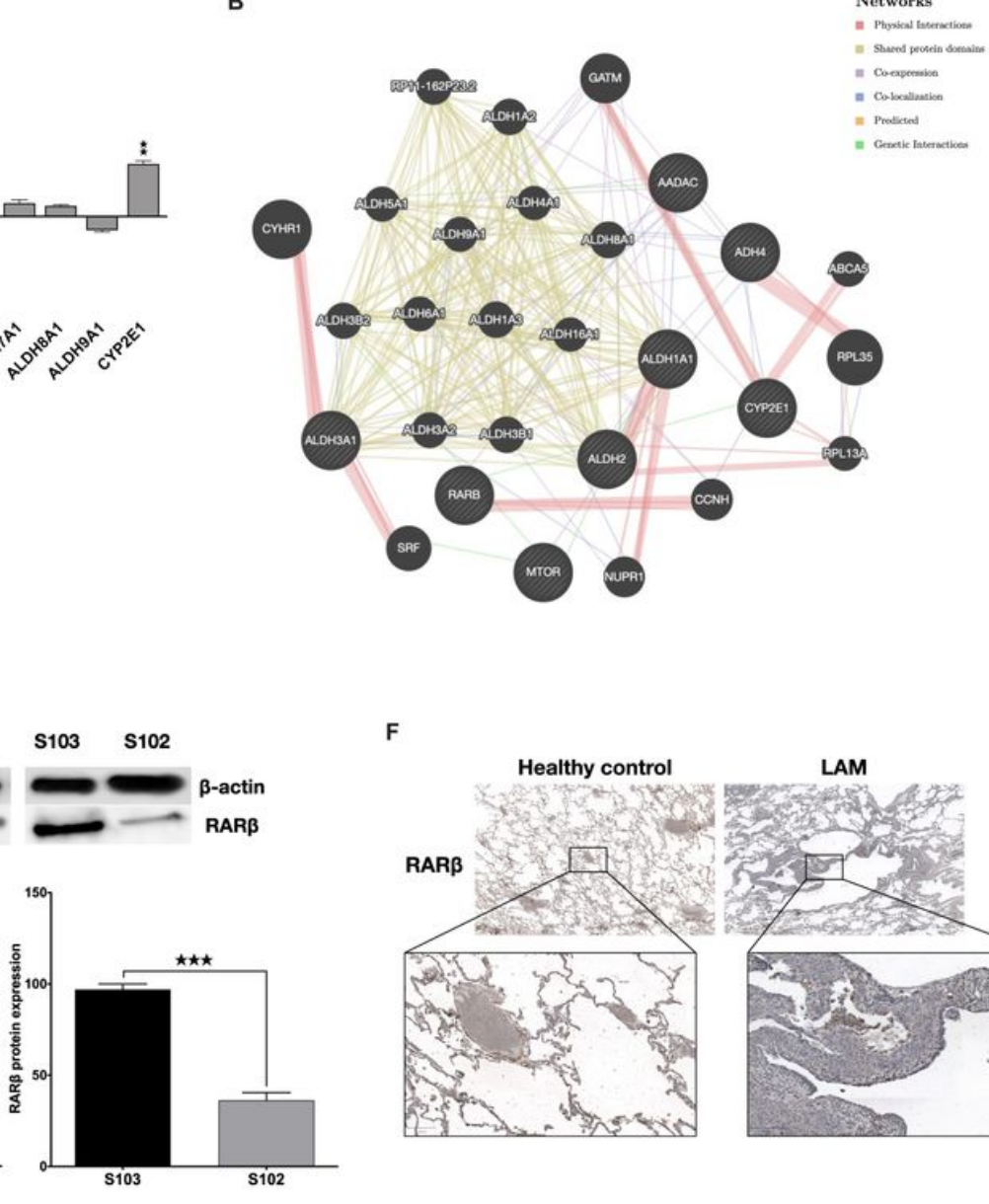

F

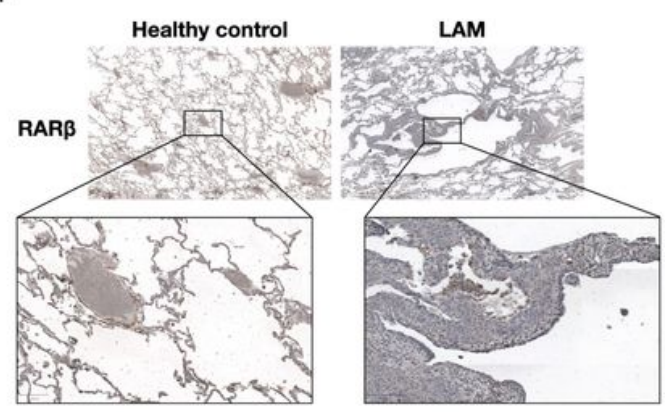

Figure 1

Metabolic enzyme and retinoic acid receptor expression. A) Human metabolic enzyme RT array analysis of S102 (TSC-/-) compared to S103 (TSC+/+) control. The figure presents Log RQ \pm of $n=3$ replicates (ttest). B) Predictive analysis of co-expression, physical interactions between metabolic enzymes and RA in TSC mutant diseases groups using GeneMANIA database. C) Gene expression of enzymes involved in vitamin A metabolism measured in LAM cell lines compared to primary healthy controls (BSMC and NHLF, 1:1). Data are presented as mean LogRQ \pm SEM compared to untreated control. Significant 


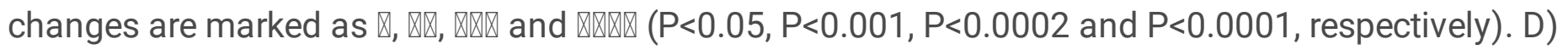
RAR $\beta$ immunofluorescent staining (RAR $\beta$ green, nuclei blue, magnification $\times 40$, size bar $28-40 \mu \mathrm{m}$ ). E) Western blot analysis of RAR $\beta$ protein levels in LAM cell lines and control cells (NHLF and BSMC). WB protein expression levels were quantified by ImageJ and are presented as percentage compared to

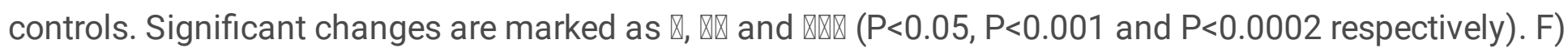
RAR $\beta$ immunohistochemistry of a representative pair of primary LAM lung sections and healthy lung controls (size bar 100-500 $\mu \mathrm{m}),(n=6)$.

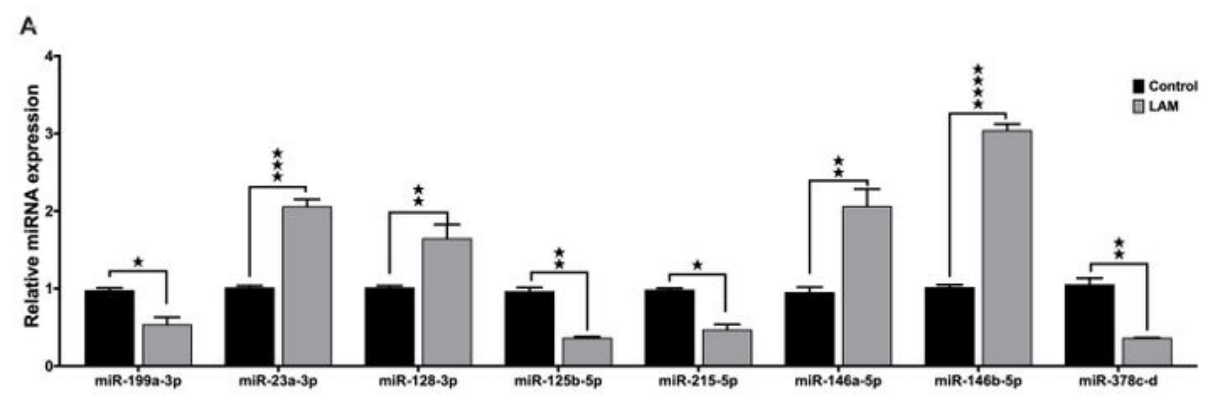

B

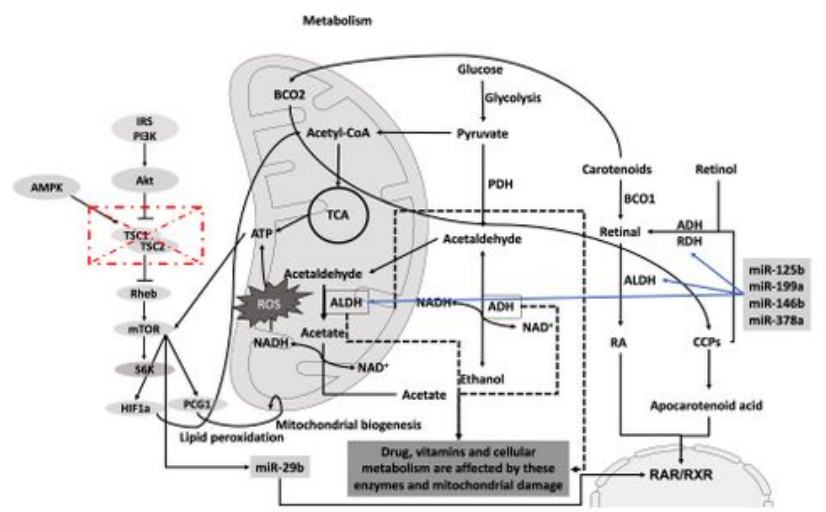

C

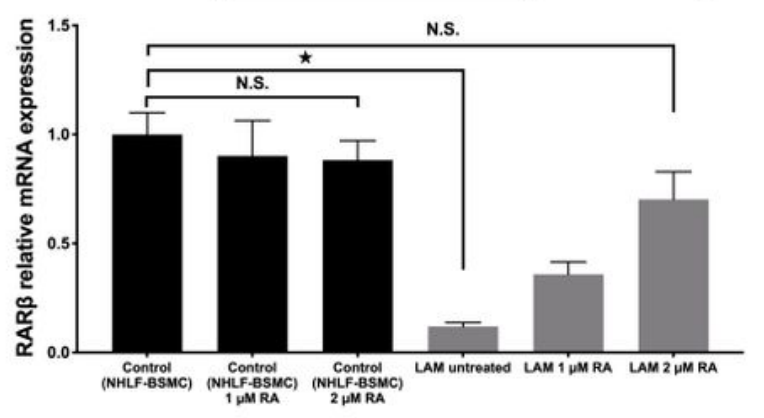

D

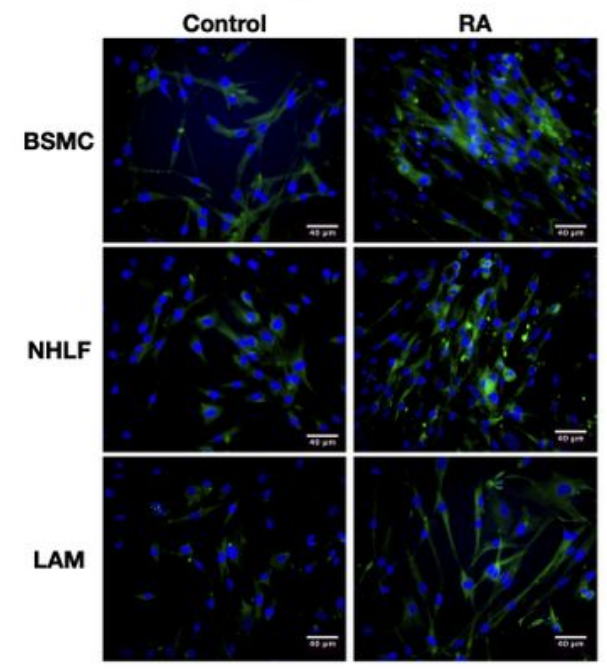


Figure 2

miRNA expression regulating metabolic enzymes in retinol production and restoration of RAR $\beta$ expression by RA. A) miRNAs that are involved in metabolic activity of cells were detected with Quantstudio $12 \mathrm{~K}$ flex. Results are presented as fold change vs. control \pm SEM. Significant changes are

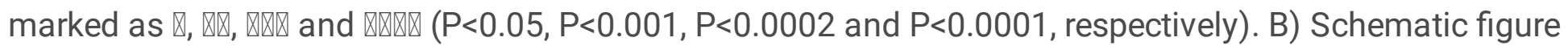
explaining the connections between different pathways, TSC-mTOR, metabolic enzymes, energy production and miRNA regulation. C) mRNA expression levels of RAR $\beta$ are significantly increased in LAM cell lines $(n=4)$ compared to controls BSMC and NHLF after $2 \mu M$ RA treatment for $24 \mathrm{~h}$. Significant

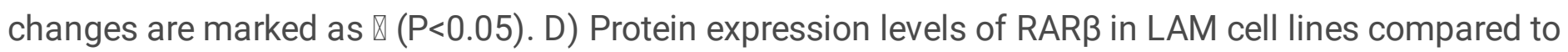
controls (BSMC and NHLF) after $2 \mu \mathrm{M}$ RA treatment for $24 \mathrm{~h}$. (RAR $\beta$ green, DAPI blue, magnification 40x, size-bar $40 \mu \mathrm{m}$ ).

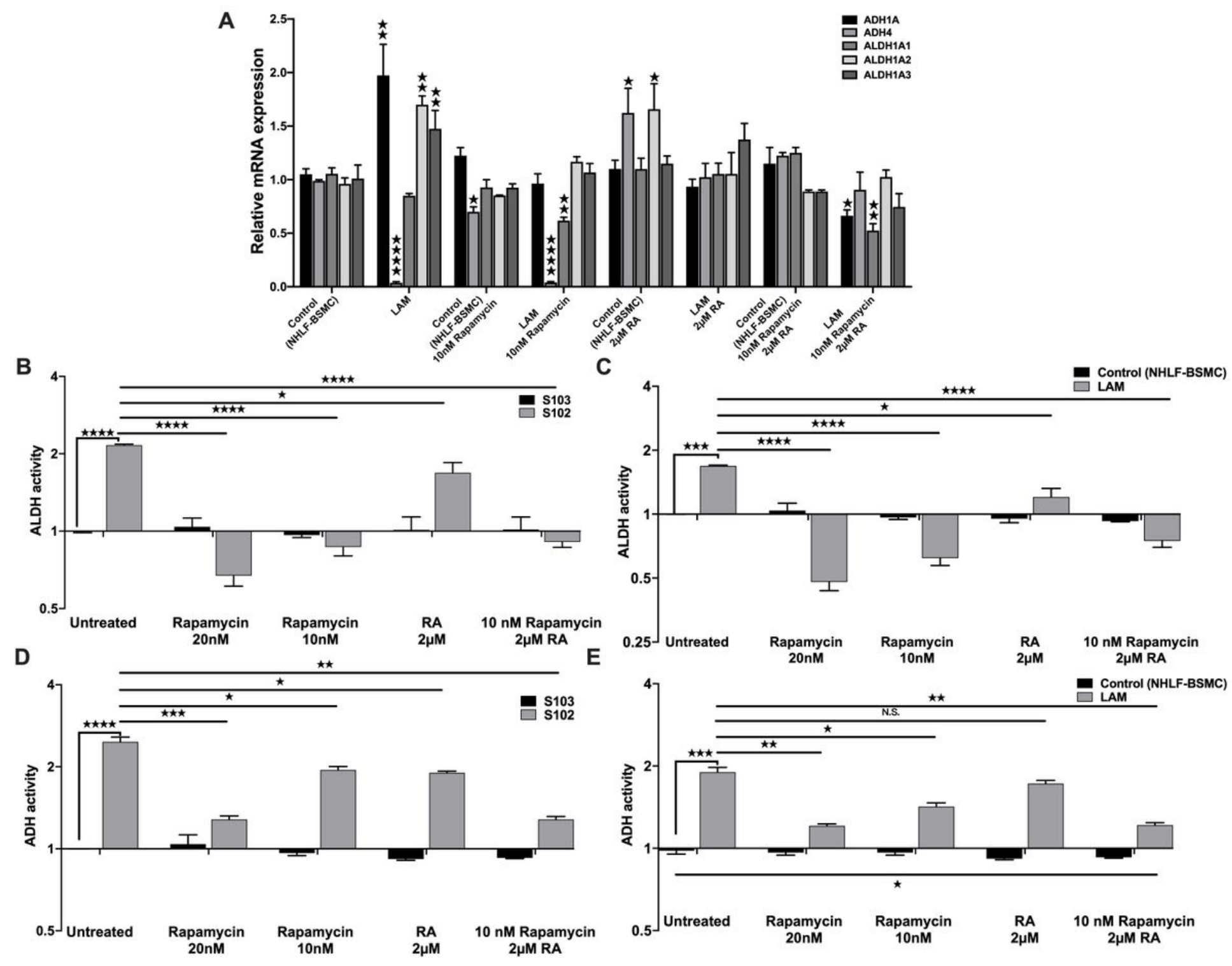

Figure 3 
The effect of RA on ALDH and ADH mRNA and enzyme activity levels. A) Gene expression levels of ALDH and ADH enzymes involved in RA metabolism following RA $(2 \mu \mathrm{M})$ treatment of LAM cell lines compared to controls (BSMC and NHLF). Data are presented as mean LogRQ \pm SEM compared to untreated controls. B) ALDH fold changes in S102 cell lines compared to untreated S103 \pm technical error of replicates (t-test) or fold of changes compared to untreated control \pm SEM (ANOVA). Significant changes

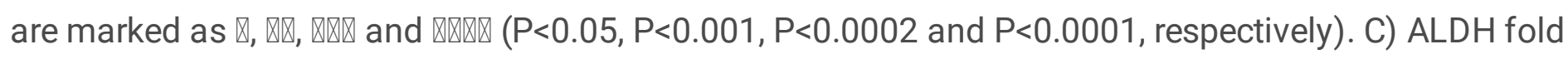
changes in LAM cell lines compared to untreated BSMC and NHLF (1:1) \pm technical error of replicates ( $t$ test) or fold of changes compared to untreated control \pm SEM (ANOVA). Significant changes are marked

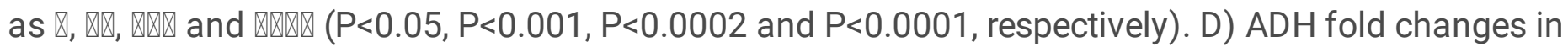
S102 cell lines compared to untreated $\mathrm{S} 103 \pm$ technical (t-test) or fold of changes compared to control

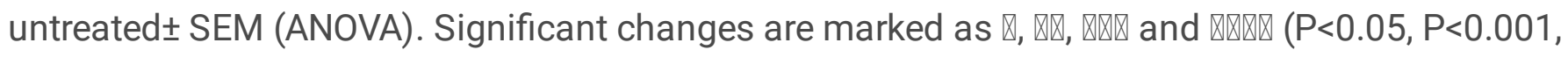
$P<0.0002$ and $P<0.0001$, respectively). E) $A D H$ fold changes in LAM cell lines compared to untreated BSMC and NHLF (1:1) \pm technical error of replicates (t-test) or fold of changes compared to control

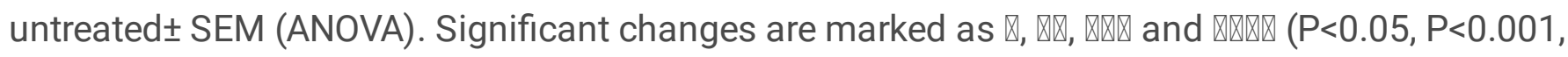
$P<0.0002$ and $P<0.0001$, respectively). 


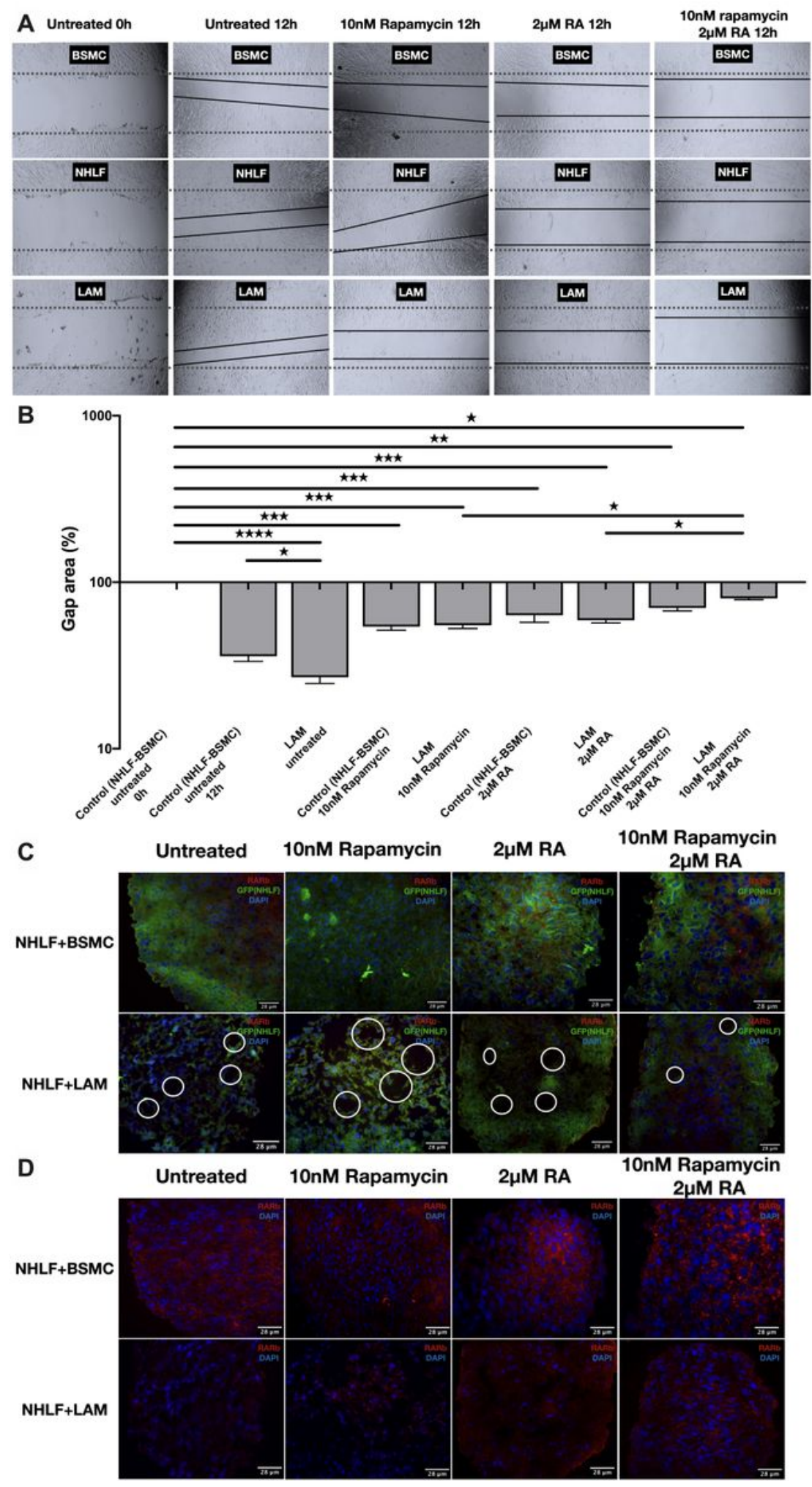

\section{Figure 4}

RA reduces cells migration capability in TSC-deficient cells. A) Wound healing scratch assay following 10 $\mathrm{nM}$ rapamycin and/or $2 \mu \mathrm{M}$ RA treatment for $24 \mathrm{~h}$. B) Wound gap area quantification, data are presented as gap area (\%) compared to untreated control (BSMC and NHLF, 1:1) as 100\%. Differences in gap

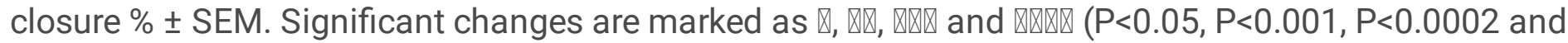
$\mathrm{P}<0.0001$, respectively). C) Empty sac formation in NHLF-LAM co-cultures compared to NHLF-BSMC co- 
cultures in the presence or absence of $10 \mathrm{nM}$ rapamycin and/or $2 \mu \mathrm{M}$ RA treatment (24 h). Empty sac formation is marked with white circles in the staining where RAR $\beta$ is red, NHLF-GFP is green and the nucleus is stained by DAPI (blue). B) RAR $\beta$ expression in 3D co-cultures after $10 \mathrm{nM}$ rapamycin and/or 2 $\mu \mathrm{M}$ RA treatment for $24 \mathrm{~h}$. RAR $\beta$ is red, NHLF-GFP is green and the nucleus is stained by DAPI (blue). Magnification 40x, size-bar $28 \mu \mathrm{m}$.

\section{Supplementary Files}

This is a list of supplementary files associated with this preprint. Click to download.

- S.Figures.pdf 